\title{
Influence of Socioeconomic Status on the Association between Prenatal Periodontal Care and Preterm Low Birthweight Delivery: A Population-Based Case-Control Study
}

\author{
Vivian Chia-Rong Hsieh ${ }^{1}$, Ya-Hui Yang ${ }^{2}$, Saou-Hsing Liou ${ }^{3}$, Chung-Yi Li ${ }^{4}$ and \\ Trong-Neng $\mathrm{Wu}^{5}$
}

${ }^{1}$ Department of Public Health, College of Public Health, China Medical University, Taichung, Taiwan; Division of Environmental Health and Occupational Medicine, National Health Research Institutes, Zhunan, Miaoli County, Taiwan

${ }^{2}$ Department of Occupational Safety and Hygiene, School of Environmental and Life Sciences, Fooyin University, Kaohsiung, Taiwan

${ }^{3}$ Division of Environmental Health and Occupational Medicine, National Health Research Institutes, Zhunan, Miaoli County, Taiwan

${ }^{4}$ Department of Public Health, College of Medicine, National Cheng Kung University, Tainan, Taiwan

${ }^{5}$ Graduate Institute of Biostatistics, College of Public Health, China Medical University and Hospital, Taichung, Taiwan; Division of Environmental Health and Occupational Medicine, National Health Research Institutes, Zhunan, Miaoli County, Taiwan

Correspondence should be addressed to: Trong-Neng Wu; E: tnwu@mail.cmu.edu.tw

Received 5 August 2013; Accepted 1 October 2013; Published 31 December 2013

Academic Editor: Shakila Zaman

Copyright (C) 2014 Vivian Chia-Rong Hsieh, Ya-Hui Yang, Saou-Hsing Liou, Chung-Yi Li and Trong-Neng Wu. Distributed under Creative Commons CC-BY 3.0

\begin{abstract}
We report the effect of in-pregnancy periodontal care intensity on the risk of preterm low birthweight (PLBW) births, and the role of household income in this association. Only a few studies have examined the role of socioeconomic status (SES) in the relationship between prenatal periodontal care and risk of PLBW delivery under universal health care. This study adopted a population-based, case-control study using medical claims data in Taiwan. Mothers of PLBW and non-PLBW babies with livebirth deliveries between 2001 and 2005 (cases, n=20430 and controls, $n=81573$, respectively) were identified using the National Health Insurance Research Database (NHIRD) and matched. Periodontal disease and disease-specific (gingivitis and periodontitis) dental service utilization was calculated during gestational period and analyzed for its association with risk for PLBW delivery. Household income as proxy for SES was tested for modification effect. Prevalence of periodontitis and gingivitis was significantly higher
\end{abstract}

Cite this Article as: Vivian Chia-Rong Hsieh, Ya-Hui Yang, Saou-Hsing Liou, Chung-Yi Li and Trong-Neng Wu (2014), "Influence of Socioeconomic Status on the Association between Prenatal Periodontal Care and Preterm Low Birthweight Delivery: A Population-Based Case-Control Study," Journal of Research and Practice in Dentistry, Vol. 2014 (2014), Article ID 469747, DOI: 10.5171/2014.469747 
in cases than in controls. Women who had these inflammatory conditions and sought treatment for either condition more frequently in gestation were at significantly lower risk for PLBW deliveries (periodontitis AOR: 0.757, 95\% CI: 0.689-0.832; gingivitis AOR: 0.684, 95\% CI: 0.582-0.805). Moreover, household income did not appear to be a significant effect modifier in the association between in-pregnancy periodontal/gingival care and PLBW delivery amongst study subjects. We found evidence that the intensity of disease-specific dental care during pregnancy acts as independent risk factor for PLBW delivery for periodontal and gingival women, and that the modification effect by SES was not evident under the context of universal health care system.

Keywords: Periodontal diseases, premature birth, socioeconomic factors, universal health care.

\section{Introduction}

Oral health of mothers has increasingly drawn attention in its link to neonatal outcomes. Periodontitis is a common oral inflammatory disease caused by poor or incorrect toothbrushing, irregular biting, stress and endocrine changes during pubertal or pregnancy period (Lai et al, 2008; Teng et al, 2003). This Gram-negative chronic anaerobic infection of the periodontium in oral cavity has been associated with preterm births as a result of an inflammatory cascade triggered by pathogenic microorganisms inducing physiologic changes in the uterine cavity that promote preterm delivery (Buduneli et al, 2005; Fardini et al, 2010; McGaw, 2002; Offenbacher et al, 1996).

The 2005 national health statistics in Taiwan revealed that roughly $30.6 \%$ of population aged between 16-64 years enrolled in the country's National Health Insurance (NHI) system (NHI enrollment rate was $98.0 \%$ in 2005) had health service utilization associated with either gingival or periodontal disease (Department of Health, 2005). These inflammatory conditions account for dental problems in a vast proportion of adult population in the country, and many of its related health adversities can be avoided if the diseases are readily identified and treated (Lai et al, 2008). Moreover, poor oral health in young adults is associated with chronic diseases, which for females, could translate intergenerationally to their offspring.

Conversely, there are investigations which failed to detect an association between periodontal disease and preterm or low birth weight (Buduneli et al, 2005; Vettore et al, 2008). There are also results from studies suggesting that periodontal care patterns or treatment of the disease during pregnancy is not associated with changes in the risk for LBW delivery (Hujoel et al, 2006; Michalowicz et al, 2006; Oliveira et al, 2011). Recent supporting meta-analyses further illustrate that treatment with scaling and root planing in this period is not protective statistically (Fogacci et al, 2011; Polyzos et al, 2010).

Moreover, income inequality has been associated with poorer health through poorer health practice or lacking of resources (Lynch et al, 2000). Lopez et al (2006) discovered social gradient present in adolescent periodontal disease which is to be linked with intermediate actors like toothbrushing and dental visit frequency. Patrick et al. (2006) demonstrated the social disparities in oral health and differential use of dental services via various sociocultural mechanisms, one specifically related to SES. Just as other developed countries, Taiwan's health system, since its implementation in 1995, aims to provide adequate and efficient health services to the population while controlling health spending within acceptable limits. It is intended to enhance horizontal equity of healthcare services (which range from western medicine, traditional Chinese medicine to dental services) for the socioeconomically-disadvantaged groups, and to avert their chance of going into financial turmoil due to unaffordable health costs. 
Only a few studies, however, have looked into the role of SES in the association between disease-specific dental utilization in pregnancy and preterm low birthweight (PLBW) delivery under this context. The purpose of this study is two-fold: first, we will examine the relationship between maternal periodontitis/gingivitis and PLBW births; second; we aim to explore the influence of dental care intensity for periodontal and gingival women during pregnancy on PLBW delivery, and whether differential household income acts as a moderator in this association in a universal health care system.

\section{Methods}

\section{Data Source}

We adopted a population-based, matched case-control design using the medical claims database of Taiwan's single-payer NHI. The National Health Insurance Research Database (NHIRD) was our source of data which contains detailed claims and reimbursement records of approximately $98 \%$ of nation's population and $78 \%$ of medical care facilities contracted with the NHI in 2005. Numerous studies have been published with NHIRD in the areas of pediatrics, obstetrics, and gynecology (Lin et al, 2004; Liu et al, 2008). These claim-based outpatient and inpatient data cover basic dental care such as periodontal treatments, which allow for a rare but valid platform to study trends in oral health of our population. This voluntary periodontal intervention subsidized by the NHI typically includes plaque control instructions, scaling, and root planing. To prevent excessive service use, a small user-fee is levied upon each dental visit, along with copayments charged for each treatment component including the pharmaceuticals. Nevertheless, this copayment is waived for low income households.

\section{Design}

\section{Subject Selection}

From 2001-5 outpatient and inpatient medical claims, we extracted PLBW babies who had diagnostic codes containing any one of International Classification of Diseases, $9^{\text {th }}$ Revision (ICD-9-CM) codes 764, 765, V21.3 which are typically inputted by physicians after delivery. Babies born in the same period without these diagnostic codes were referred to as non-PLBW babies. With the claim-based source, we believe that PLBW newborns were unlikely to go completely undetected at the time of birth. The combined outcome of preterm and low birthweight was examined because babies born preterm consequently have a low birthweight but not necessarily vice versa.

Cases (mothers of PLBW babies) and controls (mothers of non-PLBW babies) were then identified via anonymous trace through registry for beneficiaries for their enrollment status and premium payment information. Each case was matched with four controls by subject age at delivery, baby sex, and baby birth year. A final sample consisted of 20,430 cases matched to 81,573 controls after excluding individuals who were not insurance payers of their babies, continuously insured throughout observation period, or had incomplete registry data.

\section{Exposure Definition}

For cases and controls, we defined treatment groups using ICD-9-CM codes extracted within 1 year before their delivery: gingivitis 523.0-523.2, periodontitis 523.3-523.5 (i.e., those identified with history of periodontal/gingival treatment prior to pregnancy was defined as our periodontitis/gingivitis treatment groups, respectively). Primary exposure of interest 
was subjects' periodontal and gingival care during pregnancy. We realized that the reliability of coding could be problematic for administrative-based databases, especially under the current situation where individuals who were not aware of having periodontitis/gingivitis or had low income level might not be identified. However, we believe that this problem was overcome as the NHI subsidizes bi-annual dental check-up with periodontal examination (with probing of the sulcus and radiographs) offered at dental service providers from primary care clinics to medical centers. Also, with copayments (50 New Taiwan Dollars (NTD); $1 \mathrm{USD} \approx 32.8 \mathrm{NTD}$ in 2005) waived for low-income individuals and NHI's nearly $100 \%$ coverage, we believe the financial barrier was removed and the inflammatory conditions should be readily identified and diagnosed upon regular check-ups. Details about dental service delivery and reimbursement were consulted with an experienced periodontologist.

Insurance premiums of the subjects for the year of delivery were used as a proxy for household income level, assuming that payroll-based premium paid to the NHI was directly proportionate to household income. Comorbidities were also identified within 1 year before delivery while prenatal care was calculated specifically during gestation period (37 weeks for cases; 42 for controls). ICD-9-CM codes used to classify each disease are listed in Supplementary Table 1. Multiple births were defined as more than one live birth given by the same subject within a 90-day period.

\section{Statistical Analysis}

Subjects' prenatal periodontal/gingival care was quantified as measures of frequency. To account for the variable length of observation periods, frequency density (FD) was calculated using total number of disease-related dental visits within the gestation period and expressed for a person-42 week period. Square-root transformation was performed for logistic regression models to correct for the positive skewness.
In the major analyses, we used multivariable unconditional logistic regression adjusted for matching factors (age at delivery, baby sex, baby birth year), along with multiple gestation, comorbidities, prenatal care, geographical area, and household insurance premium. To detect if a linear association existed between dental attendance and birth risk, FD was entered as a linear term. Test for interaction and effect modification by household income was conducted by first using an interaction term in a joint effect model for which $P$-values for interaction were obtained, followed by a stratified analysis with income quintiles. For all statistical analyses, $P$-value of less than 0.05 was considered to be statistically significant. All analyses were performed using SAS statistical package version 9.2 (SAS Institute, Cary, NC). This study was reviewed and granted ethics approval by the Institutional Review Board at the China Medical University Hospital.

\section{Results}

Mean age of subjects was 29.4 years for both cases and controls of this study (Table 1). Distributions for maternal age, baby's birth year, and baby sex did not differ significantly between the study groups due to matching. Of the 20,430 cases and 81,573 controls, $16.5 \% \quad(n=3,380)$ and $1.4 \% \quad(n=1,169)$ experienced multiple gestation for the latter and former groups, respectively $(P<0.0001)$. Household insurance premium was slightly higher for mothers of non-PLBW babies $(26,835 \pm 16,734$ NTD $)$ than those of PLBW babies $(25,922 \pm 16,249$ NTD; $P<0.0001)$. Value of this difference of over 900NTD per household could be equivalent to 8-10 sufficient meals for an individual in 2005 . Higher prenatal care frequency was also observed for controls $(\mathrm{P}<0.0001)$.

Prevalence of comorbidities such as DM, renal disease, hypertension, chronic lung disease, genitourinary tract infections, inflammatory disease of cervix, vagina and vulva, and smoking, alcohol, drug and substance abuse was found to be significantly higher in mothers of PLBW babies than mothers of normal babies (Table 
1). The relative higher proportion of case subjects diagnosed with gingivitis $(4.9 \%$ vs. $4.3 \%$ ) and periodontitis (12.9\% vs. $11.5 \%$ ) compared with their counterparts also reached statistical significance. The positive association between gingivitis and periodontitis in both crude and adjusted logistic regression models was also significant (Table 2).

Table 1: Characteristic Profile of Subjects by Study Group (N=102 003)

\begin{tabular}{|c|c|c|c|c|c|c|}
\hline & & \multicolumn{2}{|c|}{ Case } & \multicolumn{2}{|c|}{ Control } & \\
\hline & & \multicolumn{2}{|c|}{$n=20430$} & \multicolumn{2}{|c|}{$n=81573$} & P-value \\
\hline & & $\mathrm{n}$ & $\%$ & $\mathrm{n}$ & $\%$ & \\
\hline \multicolumn{7}{|l|}{ General characteristics } \\
\hline \multirow[t]{2}{*}{ Age (years) } & & & & & & 0.85 \\
\hline & Mean \pm SD & \multicolumn{2}{|c|}{$29.4 \pm 5.3$} & \multicolumn{2}{|c|}{$29.4 \pm 5.3$} & \\
\hline \multirow[t]{6}{*}{ Baby birth year } & & & & & & 1.00 \\
\hline & 2001 & 5342 & 26.2 & 21335 & 26.2 & \\
\hline & 2002 & 4837 & 23.7 & 19337 & 23.7 & \\
\hline & 2003 & 4883 & 23.9 & 19495 & 23.9 & \\
\hline & 2004 & 3101 & 15.2 & 12363 & 15.2 & \\
\hline & 2005 & 2267 & 11.1 & 9043 & 11.1 & \\
\hline \multirow[t]{3}{*}{ Baby sex } & & & & & & 0.98 \\
\hline & Male & 11192 & 54.8 & 44694 & 54.8 & \\
\hline & Female & 9238 & 45.2 & 36879 & 45.2 & \\
\hline \multirow[t]{4}{*}{ Multiple births } & & & & & & $<0.000$ \\
\hline & & & & & & 1 \\
\hline & No & 17050 & 83.5 & 80404 & 98.6 & \\
\hline & Yes & 3380 & 16.5 & 1169 & 1.4 & \\
\hline \multirow[t]{7}{*}{ Geographical area } & & & & & & 0.02 \\
\hline & North & 9869 & 48.3 & 39468 & 48.4 & \\
\hline & Central & 4733 & 23.2 & 19496 & 23.9 & \\
\hline & South & 4941 & 24.2 & 19013 & 23.3 & \\
\hline & East & 722 & 3.5 & 2817 & 3.5 & \\
\hline & Islands & 114 & 0.6 & 540 & 0.7 & \\
\hline & Others & 51 & 0.3 & 239 & 0.3 & \\
\hline \multirow{3}{*}{$\begin{array}{l}\text { Household insurance premium } \\
\left(\mathrm{NTD}^{\mathrm{a}}\right)\end{array}$} & & & & & & $<0.000$ \\
\hline & & & & & & 1 \\
\hline & Mean \pm SD & \multicolumn{2}{|c|}{$25922 \pm 16249$} & \multicolumn{2}{|c|}{$26835 \pm 16734$} & \\
\hline
\end{tabular}

Vivian Chia-Rong Hsieh, Ya-Hui Yang, Saou-Hsing Liou, Chung-Yi Li and Trong-Neng Wu (2014), Journal of Research and Practice in Dentistry, DOI: 10.5171/2014.469747 


\begin{tabular}{|c|c|c|c|c|c|c|}
\hline Prenatal care ${ }^{b}$ & & & & & & $\begin{array}{c}<0.000 \\
1\end{array}$ \\
\hline & Mean \pm SD & 4.12 & 4.05 & 4.41 & 4.47 & \\
\hline Comorbidities & & & & & & \\
\hline Diabetes mellitus & & & & & & $<0.000$ \\
\hline & & & & & & 1 \\
\hline & No & 18785 & 91.9 & 76972 & 94.4 & \\
\hline & Yes & 1645 & 8.1 & 4601 & 5.6 & \\
\hline Renal disease & & & & & & $<0.000$ \\
\hline & & & & & & 1 \\
\hline & No & 20359 & 99.6 & 81494 & 99.9 & \\
\hline & Yes & 71 & 0.4 & 79 & 0.1 & \\
\hline Hypertension & & & & & & $<0.000$ \\
\hline & & & & & & 1 \\
\hline & No & 18642 & 91.2 & 79958 & 98.0 & \\
\hline & Yes & 1788 & 8.8 & 1615 & 2.0 & \\
\hline Chronic lung disease & & & & & & 0.02 \\
\hline & No & 20064 & 98.2 & 80305 & 98.5 & \\
\hline & Yes & 366 & 1.8 & 1268 & 1.5 & \\
\hline Genitourinary tract infections & & & & & & $<0.000$ \\
\hline & & & & & & 1 \\
\hline & No & 20198 & 98.9 & 81199 & 99.5 & \\
\hline & Yes & 232 & 1.1 & 374 & 0.5 & \\
\hline Inflammatory disease of cervix, & & & & & & $<0.000$ \\
\hline vagina, and vulva & & & & & & 1 \\
\hline & No & 14827 & 72.6 & 62307 & 76.4 & \\
\hline & Yes & 5603 & 27.4 & 19266 & 23.6 & \\
\hline Smoking, alcohol, drug and & & & & & & 0.0005 \\
\hline & No & 20288 & 99.3 & 81168 & 99.5 & \\
\hline & Yes & 142 & 0.7 & 405 & 0.5 & \\
\hline Gingivitis & & & & & & 0.0007 \\
\hline & No & 19439 & 95.1 & 78060 & 95.7 & \\
\hline & Yes & 991 & 4.9 & 3513 & 4.3 & \\
\hline Periodontitis & & & & & & $<0.000$ \\
\hline
\end{tabular}

Vivian Chia-Rong Hsieh, Ya-Hui Yang, Saou-Hsing Liou, Chung-Yi Li and Trong-Neng Wu (2014), Journal of Research and Practice in Dentistry, DOI: 10.5171/2014.469747 


\begin{tabular}{|l|r|c|c|c|c|c|}
\hline & & & & & & 1 \\
\hline & No & 17797 & 87.1 & 72226 & 88.5 & \\
\hline & Yes & 2633 & 12.9 & 9347 & 11.5 & \\
\hline a 1 USD=32.8NTD (2005). \\
$\begin{array}{l}\text { b Number of prenatal care visits converted to density as expressed for a 42-week observation } \\
\text { period. }\end{array}$ \\
\hline c Includes tobacco use disorder. \\
\hline Abbreviations: NTD = New Taiwan Dollars; USD = US Dollars. \\
\hline
\end{tabular}

\section{Supplementary Table1: Delivery Outcomes and Maternal Diseases Classification and} Associated ICD-9 Codes a

\begin{tabular}{|l|l|}
\hline Category & ICD-9 codes \\
\hline Delivery outcomes & \\
\hline Preterm or low birth weight & $764,765, \mathrm{~V} 21.3$ \\
\hline Oral disease & \\
\hline Gingivitis & $523.0-523.2$ \\
\hline Periodontitis & $523.3-523.5$ \\
\hline Maternal comorbidities & $250,648.0,648.8$ \\
\hline Diabetes mellitus & $401-402,405,642$ \\
\hline Hypertension & $582,585-586$ \\
\hline Chronic renal disease (inc. ESRD, severe renal disease) & $490,491,492,496$ \\
\hline Chronic disease of the lung & $291,303-304,386$, \\
\hline Smoking, alcohol, drug and substance abuse & $648.3,649.0$ \\
\hline Infections of genitourinary tract & 646.6 \\
\hline Inflammatory disease of cervix, vagina, and vulva & 616 \\
\hline
\end{tabular}

a International Classification of Diseases, Ninth Revision, Clinical Modification (ICD-9-CM), Sixth Edition, U.S.

Department of Health and Human Services. Centers for Disease Control and Prevention, National Center for Health Statistics. Hyattsville, MD.

Subjects' household insurance premium was classified into income quintiles based on control group's premium distribution in Figure 1 (Q5 (top 20\%): >=NTD38,200; Q4: NTD25,200-38,199; Q3: NTD19,200-25,199; Q2: NTD17,280-19,199; Q1 (bottom 20\%): $\mathrm{NTD}<17,280$ ). In intra-group comparison, higher prenatal disease-specific dental care was generally detected in lower income groups for both groups; a gradient across income quintiles was observed in an inverse relationship between mean prenatal dental visit $\mathrm{FD}$ and income class. Between study groups, controls had relatively higher gingival and periodontal care in pregnancy as compared with cases on average. 

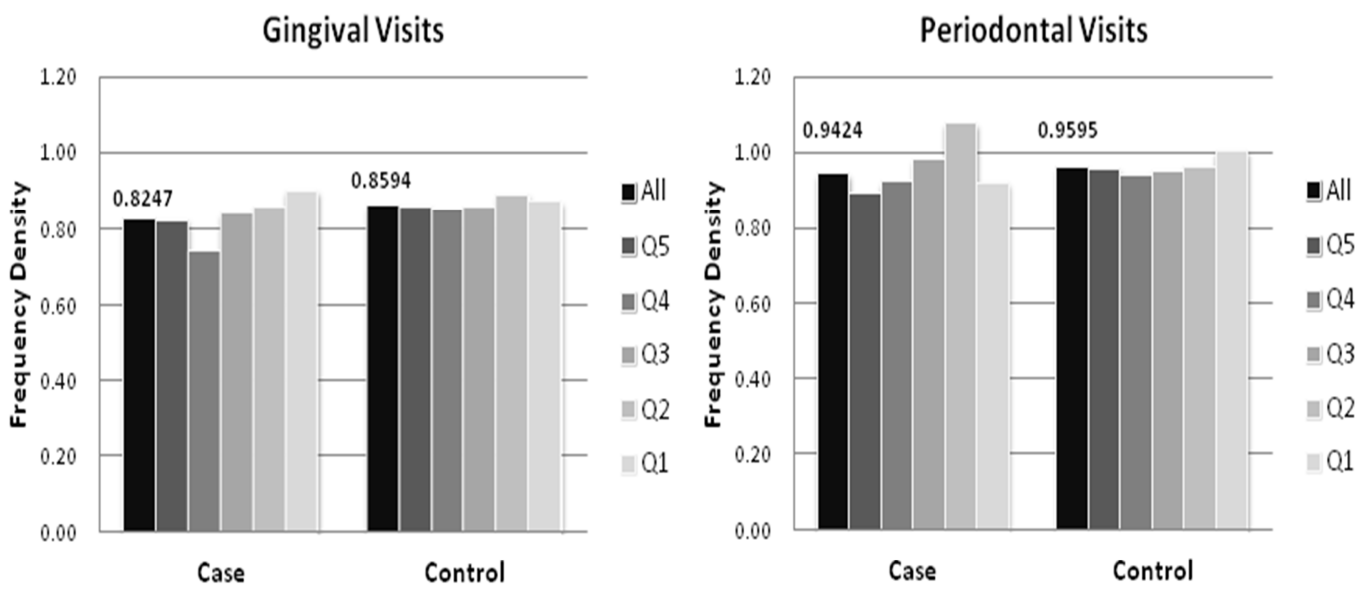

Figure 1 Gradient in dental visit frequency density during pregnancy across different household income quintiles according to disease type by study group. Left: gingival visits; Right: periodontal visits. (All: average, Q5: top income quintile, Q1: bottom income quintile; Q5:Q1 ratios not shown.)

Mean and median visit FD for gingival care before square-root transformation was 0.040 and 0 (range: 4.541) in cases, and 0.037 and 0 (range: 4.000) in controls, respectively. For periodontal care, mean and median FD was 0.121 and 0 (range: 15.892) in cases, and 0.110 and 0 (range: 9.000) in controls, respectively.

Table 2 examines the association between risk for PLBW deliveries and the intensity of disease-specific dental care sought by the subjects during pregnancy. Women who sought treatment for periodontitis or gingivitis more frequently in gestation were at significantly lower risk for PLBW deliveries. This was shown in both crude and adjusted models. After controlling for multiple covariates as indicated in Table 2, higher prenatal periodontal care FD was associated with a lower risk for PLBW delivery (adjusted odds ratio (AOR): 0.757; 95\% confidence interval (CI): 0.689-0.832, $\mathrm{P}<0.0001)$. Same pattern was observed with prenatal gingival care (AOR: 0.684; 95\% CI: 0.582-0.805, $\mathrm{P}<0.0001$ ). 
Table 2: Periodontal Diseases, Disease-Specific Dental Service Utilization in Pregnancy and Relative Risks for Preterm Low Birth Weight (PLBW) Delivery

\begin{tabular}{|c|c|c|c|c|c|c|}
\hline & \multicolumn{3}{|c|}{ Crude model } & \multicolumn{3}{|c|}{ Adjusted model ${ }^{b}$} \\
\hline & OR & $95 \% \mathrm{CI}$ & P-value & OR & $95 \% \mathrm{CI}$ & P-value \\
\hline \multicolumn{7}{|l|}{ Periodontal disease } \\
\hline Gingivitis & 1.133 & $1.054-1.218$ & 0.0007 & 1.162 & $1.073-1.258$ & 0.0002 \\
\hline Periodontitis & 1.143 & $1.092-1.197$ & $<0.0001$ & 1.178 & $1.118-1.242$ & $<0.0001$ \\
\hline \multicolumn{7}{|c|}{ Visit frequency density ${ }^{a}$} \\
\hline Gingival care & 0.694 & $0.599-0.803$ & $<0.0001$ & 0.684 & $0.582-0.805$ & $<0.0001$ \\
\hline Periodontal care & 0.771 & $0.708-0.841$ & $<0.0001$ & 0.757 & $0.689-0.832$ & $<0.0001$ \\
\hline \multicolumn{7}{|c|}{ Note: $\mathrm{OR}=$ odds ratio; $\mathrm{CI}$ = confidence interval. } \\
\hline \multicolumn{7}{|c|}{ a Frequency density of visits have been square-root transformed and presented in the models as continuous variables. } \\
\hline \multicolumn{7}{|c|}{$\begin{array}{l}\text { b Models adjusted for: age at delivery, baby sex, baby birth year, multiple birth, geographical area, household income, } \\
\text { frequency density of prenatal care visits, and comorbidities (diabetes mellitus, renal disease, hypertension, chronic } \\
\text { lung disease, genitourinary tract infections, inflammatory disease of cervix, vagina, and vulva, and smoking, alcohol, } \\
\text { drug and substance abuse). }\end{array}$} \\
\hline
\end{tabular}

\section{Supplementary Table 2: Periodontal Diseases, Disease-Specific Dental Service Utilization in Pregnancy and Relative Risks for Preterm Low Birth Weight (PLBW) Delivery (Singleton Births Only)}

\begin{tabular}{|c|c|c|c|c|c|c|c|}
\hline & \multicolumn{3}{|c|}{ Crude model } & \multicolumn{3}{c|}{ Adjusted model $^{\text {b }}$} \\
\hline & OR & $95 \% \mathrm{CI}$ & P-value & OR & $95 \% \mathrm{CI}$ & P-value \\
\hline Periodontal disease & & & & & & & \\
\hline Gingivitis & 1.090 & $1.008-1.180$ & 0.0316 & & 1.163 & $1.071-1.263$ & 0.0003 \\
\hline Periodontitis & 1.105 & $1.051-1.162$ & $<0.0001$ & & 1.181 & $1.118-1.247$ & $<0.0001$ \\
\hline Visit frequency density & & & & & & & \\
\hline Gingival care & 0.700 & $0.596-0.822$ & $<0.0001$ & & 0.693 & $0.586-0.820$ & $<0.0001$ \\
\hline Periodontal care & 0.784 & $0.713-0.861$ & $<0.0001$ & & 0.766 & $0.695-0.844$ & $<0.0001$ \\
\hline
\end{tabular}

Note: $\mathrm{OR}=$ odds ratio; $\mathrm{CI}=$ confidence interval.

a Frequency density of visits have been square-root transformed and presented in the models as continuous variables.

b Models adjusted for: age at delivery, baby sex, baby birth year, multiple birth, geographical area, household income, frequency density of prenatal care visits, and comorbidities (diabetes mellitus, renal disease, hypertension, chronic lung disease, genitourinary tract infections, inflammatory disease of cervix, vagina, and vulva, and smoking, alcohol, drug and substance abuse).

\section{Effect Modification}

To examine if household income exerted a modification effect, an interaction term with household insurance premium and prenatal dental care FD was introduced. Household income did not appear to be a significant effect modifier in the association between 
in-pregnancy periodontal/gingival care and PLBW delivery amongst study subjects (P for interaction: 0.07 (gingival care) and 0.76 (periodontal care) (Table 3). This suggests that the effect of prenatal disease-specific care on delivery outcome is ubiquitously beneficial across income groups.

Table 3: Modification by Household Income on the Effect of Dental Visit Frequency Density in Pregnancy on Preterm Low Birth Weight (PLBW) Delivery

\begin{tabular}{|c|c|c|c|c|}
\hline Frequency density ${ }^{\mathrm{a}}$ & $\begin{array}{c}\text { Household } \\
\text { income level }\end{array}$ & $\mathrm{AOR}^{\mathrm{c}}$ & $95 \%$ CI & $\begin{array}{l}P \text { for } \\
\text { interaction }^{\mathrm{d}}\end{array}$ \\
\hline \multirow[t]{6}{*}{ Gingival care } & & & & 0.07 \\
\hline & Q5 & 0.71 & $0.50-1.02$ & \\
\hline & Q4 & $0.52^{* *}$ & $0.37-0.74$ & \\
\hline & Q3 & $0.71^{*}$ & $0.53-0.95$ & \\
\hline & Q2 & 0.69 & $0.37-1.28$ & \\
\hline & Q1 & 0.91 & $0.60-1.38$ & \\
\hline \multirow[t]{6}{*}{ Periodontal care } & & & & 0.76 \\
\hline & Q5 & $0.67^{* *}$ & $0.56-0.81$ & \\
\hline & Q4 & $0.71^{* *}$ & $0.58-0.88$ & \\
\hline & Q3 & 0.86 & $0.73-1.02$ & \\
\hline & Q2 & 1.09 & $0.76-1.57$ & \\
\hline & Q1 & $0.64^{* *}$ & $0.50-0.81$ & \\
\hline \multicolumn{5}{|c|}{$\begin{array}{l}\text { Note: } \mathrm{AOR}=\text { adjusted odds ratio; } \mathrm{CI}=\text { confidence interval; } \mathrm{Q}=\text { quintile; } \mathrm{NTD}=\text { New Taiwan } \\
\text { Dollars. }\end{array}$} \\
\hline \multicolumn{5}{|c|}{$\begin{array}{l}\text { a Frequency density of visits have been square-root transformed and presented in the models as } \\
\text { continuous variables. }\end{array}$} \\
\hline \multicolumn{5}{|c|}{$\begin{array}{l}\text { b Income level classification - Q5: } \geqq 38200 N T D ; \text { Q4: 25200-38199NTD; Q3:19200-25199NTD; } \\
\text { Q2:17280-19199NTD; Q1:<17280NTD. }\end{array}$} \\
\hline \multicolumn{5}{|c|}{ c Model adjusted for factors as in Table 2.} \\
\hline \multicolumn{5}{|c|}{$\begin{array}{l}\text { d } P \text { for interaction: tested using interaction term including household income and frequency } \\
\text { density. }\end{array}$} \\
\hline$*: \mathrm{P}<0.05 ; * *: \mathrm{P}<0.00$ & & & & \\
\hline
\end{tabular}




\section{Supplementary Table 3: Modification by Household Income on the Effect of Dental Visit Frequency Density in Pregnancy on Preterm Low Birth Weight (PLBW) Delivery (Singleton Births Only)}

\begin{tabular}{|c|c|c|c|c|}
\hline Frequency density ${ }^{a}$ & $\begin{array}{c}\text { Household } \\
\text { income level }\end{array}$ & $\mathrm{AOR}^{\mathrm{c}}$ & $95 \%$ CI & $\begin{array}{c}P \text { for } \\
\text { interaction }^{\mathrm{d}}\end{array}$ \\
\hline \multirow[t]{6}{*}{ Gingival care } & & & & 0.20 \\
\hline & Q5 & 0.69 & $0.48-1.01$ & \\
\hline & Q4 & $0.54^{* *}$ & $0.38-0.78$ & \\
\hline & Q3 & $0.73^{*}$ & $0.54-0.98$ & \\
\hline & Q2 & 0.59 & $0.31-1.13$ & \\
\hline & Q1 & 0.93 & $0.60-1.42$ & \\
\hline \multirow[t]{6}{*}{ Periodontal care } & & & & 0.85 \\
\hline & Q5 & $0.68^{* *}$ & $0.56-0.82$ & \\
\hline & Q4 & $0.69 *$ & $0.56-0.86$ & \\
\hline & Q3 & 0.91 & $0.76-1.08$ & \\
\hline & Q2 & 1.09 & $0.75-1.59$ & \\
\hline & Q1 & $0.64^{* *}$ & $0.50-0.82$ & \\
\hline \multicolumn{5}{|c|}{$\begin{array}{l}\text { Note: } \mathrm{AOR}=\text { adjusted odds ratio; } \mathrm{CI}=\text { confidence interval; } \mathrm{Q}=\text { quintile; } \mathrm{NTD}=\text { New Taiwan } \\
\text { Dollars. }\end{array}$} \\
\hline \multicolumn{5}{|c|}{$\begin{array}{l}\text { a Frequency density of visits have been square-root transformed and presented in the models as } \\
\text { continuous variables. }\end{array}$} \\
\hline \multicolumn{5}{|c|}{$\begin{array}{l}\text { b Income level classification - Q5:ฏ38200NTD; Q4: 25200-38199NTD; Q3:19200-25199NTD; } \\
\text { Q2:17280-19199NTD; Q1:<17280NTD. }\end{array}$} \\
\hline \multicolumn{5}{|c|}{ c Model adjusted for factors as in Supplementary Table 2.} \\
\hline \multicolumn{5}{|c|}{$\begin{array}{l}\text { d } P \text { for interaction: tested using interaction term including household income and frequency } \\
\text { density. }\end{array}$} \\
\hline
\end{tabular}

\section{Discussion}

In this population-based case-control study, we found evidence that both periodontitis and gingivitis were associated with PLBW delivery. We further demonstrated that women with periodontitis or gingivitis, in spite of their income class, who received more intensive care in terms of frequency for their dental conditions during pregnancy had lower risk for PLBW delivery compared with women who received less intensive care.

Support for maternal periodontal disease acting as a significant risk factor for PLBW births has been established by numerous studies (Khader et al, 2009; Offenbacher et al, 1996; Moliterno et al, 2005). Currently, markers identified in periodontal women presenting with the risk for preterm delivery 
include prostaglandins, inflammatory cytokines, bacterial products like endotoxins and fetal adrenal cortisone - combination of which can lead to myometrial contraction and cervical ripening (Boggess et al, 2005; McGaw, 2002; Vergnes and Sixou, 2007). Hormonal change during gestation is also believed to promote gum inflammation causing destructive oral diseases like gingivitis and periodontitis (Siristatidis et al, 2006).

However, it becomes complicated when considering periodontal or gingival care for pregnant women. A general concern by the women is the negative effect of treatment such as x-ray and anesthesia on gestational outcome (Dinas et al, 2007). Dentists typically avoid or postpone surgical periodontal treatments until after delivery, except when patients exhibit severe bleeding or are incapable of ingesting food. Thus, utilization of dental services has generally observed to be low for antenatal mothers also for other reasons such as delaying treatment until delivery or not having adequate knowledge for oral health problems (Al Habashneh et al, 2005). An epidemiological study with a non-random sample in Taiwan from 2007-8 indicated that increasing age, poor toothbrushing knowledge and low socioeconomic status (SES) are potential risk factors of the disease (Lai et al, 2008). In spite of this, advantages of periodontal care during pregnancy on birth outcomes have been previously documented in clinical trials and intervention studies (Cruz et al, 2010; Jeffcoat et al, 2011; Radnai et al, 2009; Tarnnum and Faizuddin, 2007). Kim et al. (2012) proved reduced risk only for high risk pregnant women in a meta-analysis of randomized controlled trials on scaling and root planing treatment.

Unfortunately, we could not distinguish if the treatment received by our subjects was surgical or non-surgical from the available data. Nonetheless, our findings support that therapy to alleviate pain should be performed under any circumstance since stress symptoms resulting from pain could have a worse impact on the mother and fetus than the negative consequences of treatment.

Several aspects of this study design also need to be addressed for correct interpretation of results. First, one caveat of using a medical claims database was the lack of information on lifestyle and environmental risks for PLBW, like marital status, education level, smoking and diet. Smoking in women is a critical risk factor for PLBW, and lowest SES women in Taiwan have been detected with highest smoking prevalence and nicotine level (Chang, 2006). We attempted to control for this effect by adjusting for smoking, alcohol, drug and substance abuse in our analyses. Second, although diagnostic codes indicating diseases status were available and constituted our exposure measure, we were unable to extract individuals who were unaware of their dental conditions and did not seek any care despite the free dental check-ups. For women who could not afford periodontal care, however, we have minimal concern of overlooking them during the 1 year observation period since their copayments are waived and dental care is readily accessible in the nearly $100 \%$ insured population.

In this study, we illustrated that the positive influence of periodontal/gingival care intensity on time of delivery and birthweight is robust for different household-level incomes under the current health system. Higher utilization was also observed in lower income groups. This outcome is promising as the NHI appears to be reducing the utilization gradient as suggested by Cheng and Chiang (1997). This is in contrast with the results from a study by Grembowski et al (1989) done under context of a different health insurance system (i.e.., a gradient and a lower utilization in socioeconomically-disadvantaged groups). A Chilean study similarly identified a social gradient in adolescent periodontal diseases that was largely contributed by paternal income and education (López et al, 2006). Patrick and colleagues (2006) additionally discussed about bias by dentists towards low-income women as well as differential access to oral care by numerous factors including disadvantaged economic status. In 
this case, however, we did not see an effect modification by income level. Thus, we could interpret such that the quality of care is generally standardized for women across different SES groups. Since a vast proportion of dental service providers are contracted with the NHI and patients are free to choose their providers without a referral system in place, we believe that the dentists serving poor women are not different from those serving rich women in the general context.

To our knowledge, this is the one of the few studies performed under a universal health care system in which the role of household-level SES has been investigated in the association between periodontal care utilization and pregnancy outcome. Unlike previous reviews or studies with relatively smaller samples, we had in our study a large nationally-representative sample of antenatal mothers compiled within a five-year period for a clear pinpoint of the causal inference relationship. Potential confounders including maternal comorbidities were controlled for in the analyses. Reliability of data used should be high due to rigorous screening process. The application of large-sample NHIRD data should be justified as numerous studies have been published with use of the same dataset (Lin et al, 2004; Liu et al, 2008).

Poor oral health in an expectant mother concerns not only of her own health but of her children's as well. Women should be encouraged to seek dental care even during gestation. Furthermore, the effect of dental service utilization in pregnancy under universal health care is not sensitive to household income which suggests removal of financial barrier and easy accessibility to dental services are critical measures to ensuring good pregnancy outcome. This is particularly relevant in this context where everyone is entitled to the same type of services, and has the freedom to choose their own providers at times when they feel the need of medical attention (i.e. no gatekeeper system). Still, examination into the specific types of treatment and their relevant timing should be warranted in future investigation.

\section{Conclusions}

In summary, we found evidence that intensity of disease-specific dental care in pregnancy acts as independent risk factor to preterm low birthweight delivery for periodontal and gingival women, and that there was no modification effect by household income level. Women with periodontitis or gingivitis are advised to seek dental care during pregnancy for routine examination and treatment for periodontal diseases. With investigation into dental conditions that could intergenerationally translate into life-long adversity, Taiwan's universal health care system appears to be performing well in terms of averting disparities in health.

\section{Acknowledgment}

This study is based on data provided and managed by the National Health Research Institutes (NHRI) in Taiwan. We thank the NHRI for providing NHIRD and administrative support for research purposes (Project No. 98349), as well as the National Science Council (Taiwan) for the grant support (NSC 101-2314-B-039-016).

\section{References}

Al Habashneh, R., Guthmiller, J. M., Levy, S., Johnson, G. K., Squier, C., Dawson, D. V. \& Fang, Q. (2005). "Factors Related to Utilization of Dental Services during Pregnancy," Journal of Clinical Periodontology, 32 (7) 815-821.

Boggess, K. A., Moss, K., Madianos, P., Murtha, A. P., Beck, J. \& Offenbacher, S. (2005). "Fetal Immune Response to Oral Pathogens and Risk of Preterm Birth," American Journal of Obstetrics and Gynecology, 193 (3 Pt 2) 1121-1126.

Buduneli, N., Baylas, H., Buduneli, E., Türkoğlu, O., Köse, T. \& Dahlen, G. (2005). "Periodontal Infections and Pre-Term Low Birth Weight: A Case-Control Study," Journal of Clinical Periodontology, 32 (2) 174-181. 
Chang, S. L. (2006). 'A Study of Socio-Economic Class and Healthy Behavior,' Self-Rated Health in Taiwan, Masters Dissertation, Department of Sociology, National Taipei University, Taiwan.

Cheng, S. H. \& Chiang, T. L. (1997). "The Effect of Universal Health Insurance on Health Care Utilization: Results from a Natural Experiment," Journal of the American Medical Association, 278 (2) 89-93.

Cruz, S. S., Costa M. da C., Gomes-Filho, I. S., Barreto, M. L., dos Santos, C. A., Martins, A. G., Passos J. de S., De Freitas, C. O., Sampaio, F. P. \& Cerqueira Ede, M. (2010). "Periodontal Therapy for Pregnant Women and Cases of Low Birthweight: An Intervention Study," Pediatrics International, 52 (1) 57-64.

Department of Health, Executive Yuan, Republic of China. (2005). 'National Health Insurance Statistics for Outpatient and Inpatient Service Utilization in 2005,' Taipei, Department of Health, Executive Yuan, Republic of China (Taiwan).

Dinas, K., Achyropoulos, V., Hatzipantelis, E., Mavromatidis, G., Zepiridis, L., Theodoridis, T., Dovas, D., Tantanasis, T., Goutzioulis, F. \& Bontis, J. (2007). "Pregnancy and Oral Health: Utilisation of Dental Services during Pregnancy in Northern Greece," Acta Obstetricia et Gynecologica Scandinavica, 86 (8) 938-944.

Fardini, Y., Chung, P., Dumm, R., Joshi, N. \& Han, Y. W. (2010). "Transmission of Diverse Oral Bacteria to Murine Placenta: Evidence for the Oral Microbiome as a Potential Source of Intrauterine Infection," Infection and Immunity, 78 (4) 1789-1796.

Fogacci, M. F., Vettore, M. V. \& Leão, A. T. (2011). "The Effect of Periodontal Therapy on Preterm Low Birth Weight: A Meta-Analysis," Obstetrics and Gynecology, 117 (1) 153-165.

Grembowski, D., Andersen, R. M. \& Chen, M. (1989). "A Public Health Model of the Dental Care Process," Medical Care Review, 46 (4) 439-496.
Hujoel, P. P., Lydon-Rochelle, M., Robertson, P. B. \& Del Aguila, M. A. (2006). "Cessation of Periodontal Care during Pregnancy: Effect on Infant Birthweight," European Journal of Oral Sciences, 114 (1):2-7.

Jeffcoat, M., Parry, S., Sammel, M., Clothier, M., Catlin, A. \& Macones, G. (2011). "Periodontal Infection and Preterm Birth: Successful Periodontal Therapy Reduces the Risk of Preterm Birth," BJOG: An International Journal of Obstetrics \& Gynaecology, 118 (2) 250-256.

Khader, Y., Al-shishani, L., Obeidat, B., Khassawneh, M., Burgan, S., Amarin, Z. O., Alomari, M. \& Alkafajei, A. (2009). "Maternal Periodontal Status and Preterm Low Birthweight Delivery: A Case-Control Study," Archives of Gynecology and Obstetrics, 279 (2) 165-169.

Kim, A. J., Lo, A. J., Pullin, D. A., Thornton-Johnson, D. S. \& Karimbux, N. Y. (2012). "Scaling and Root Planing Treatment for Periodontitis to Reduce Preterm Birth And Low Birthweight: A Systematic Review and Meta-Analysis of Randomized Controlled Trials," Journal of Periodontology, 83 (12) 1508-1519.

Lai, H. M., Chen, H. H. \& Chang, S. H. (2008). 'Adult Periodontal Disease and Preventive Behavioral Survey in Taiwan 2007-8: Final Report,' Taipei, Bureau of Health Promotion, Department of Health, Executive Yuan, Republic of China (Taiwan).

Lin, H.- C., Sheen, T.- C., Tang, C.- H. \& Kao, S. (2004). "Association between Maternal Age and the Likelihood of a Cesarean Section: A Population-Based Multivariable Logistic Regression Analysis," Acta Obstetricia et Gynecologica Scandinavica, 83 (12) 1178-1183.

Liu, T.- C., Lin, H.- C., Chen, C.- S. \& Lee, H.- C. (2008). "Obstetrician Gender and the Likelihood of Performing a Maternal Request for a Cesarean Delivery," European Journal of Obstetrics and Gynecology and Reproductive Biology, 136 (1) 46-52. 
López, R., Fernández, O. \& Baelum, V. (2006). "Social Gradients in Periodontal Diseases among Adolescents," Community Dentistry and Oral Epidemiology, 34 (3) 184-196.

Lynch, J. W., Smith, G. D., Kaplan, G. A. \& House, J. S. (2000). "Income Inequality and Mortality: Importance to Health of Individual Income, Psychosocial Environment, or Material Conditions," British Medical Journal, 320 (7243) 1200-1204.

McGaw, T. (2002). "Periodontal Disease And Preterm Delivery of Low-Birth-Weight Infants," Journal of the Canadian Dental Association, 68 (3) 165-169.

Michalowicz, B. S., Hodges, J. S., DiAngelis, A. J., Lupo, V. R., Novak, M. J., Ferguson, J. E., Buchanan, W., Bofill, J., Papapanou, P. N., Mitchell, D. A., Matseoane, S., Tschida, P. A. \& OPT Study. (2006). "Treatment of Periodontal Disease and the Risk of Preterm Birth," New England Journal of Medicine, 355 (18) 1885-1894.

Moliterno, L. F., Monteiro, B., Figueredo, C. M. \& Fischer, R. G. (2005). “Association between Periodontitis and Low Birthweight: A Case-Control Study," Journal of Clinical Periodontology, 32 (8) 886-890.

Offenbacher, S., Katz, V., Fertik, G., Collins, J., Boyd, D., Maynor, G., McKaig, R. \& Beck, J. (1996). "Periodontal Infection as a Possible Risk Factor for Preterm Low Birth Weight," Journal of Periodontology, 67 (10 Suppl) 1103-1113.

Oliveira, A. M. S. D., de Oliveira, P. A., Cota, L. O. M., Magalhães, C. S., Moreira, A. N. \& Costa, F. O. (2011). "Periodontal Therapy and Risk for Adverse Pregnancy Outcomes," Clinical Oral Investigations, 15 (5) 609-615.

Patrick, D. L., Lee, R. S. Y., Nucci, M., Grembowski, D., Jolles, C. Z. \& Milgrom, P. (2006). "Reducing oral Health Disparities: A Focus on Social and Culture Determinants," BMC Oral Health, 6 Suppl 1:S4.
Polyzos, N. P., Polyzos, I. P., Zavos, A., Valachis, A., Mauri, D., Papanikolaou, E. G., Tzioras, S., Weber, D. \& Messinis, I. E. (2010). “Obstetric Outcomes after Treatment of Periodontal Disease during Pregnancy: Systematic Review and Meta-Analysis," British Medical Journal, 341:c7017.

Radnai, M., Pál, A., Novák, T., Urbán, E., Eller, J. \& Gorzó, I. (2009). "Benefits of Periodontal Therapy when Preterm Birth Threatens," Journal of Dental Research, 88 (3) 280-284.

Siristatidis, C., Nisiotakis, C., Zokaris, N., Chrelias, C., Iakovidou, H. \& Salamalekis, E. (2006). "Hormonal Alterations in Gum Disease Leading to Preterm Labor," Archives of Gynecology and Obstetrics, 274 (1) 13-18.

Tarannum, F. \& Faizuddin, M. (2007). "Effect of Periodontal Therapy on Pregnancy Outcome in Women Affected by Periodontitis," Journal of Periodontology, 78 (11) 2095-2103.

Teng, H. C., Lee, C. H., Hung, H. C., Tsai, C. C., Chang, Y. Y., Yang, Y. H., Lu, C. T., Yen, Y. Y. \& Wu, Y. M. (2003). "Lifestyle and Psychosocial Factors Associated with Chronic Periodontitis in Taiwanese Adults," Journal of Periodontology, 74 (8) 1169-1175.

Vergnes, J. N. \& Sixou, M. (2007). “Preterm Low Birth Weight and Maternal Periodontal Status: A Meta-Analysis," American Journal of Obstetrics and Gynecology, 196 (2) 135.e1-e7.

Vettore, M. V., doC. Leal, Leão, A. T., Da Silva, A. M. M., Lamarca, G. A. \& Sheiham, A. (2008). "The Relationship between Periodontitis and Preterm Low Birthweight," Journal of Dental Research, 87 (1) 73-78. 